\title{
Beckett : un monde de vieillards
}

Samuel Beckett: An Old Men's World

Marie-Claude Hubert

\section{OpenEdition}

Journals

Édition électronique

URL : https://journals.openedition.org/recherchestravaux/733

DOI : 10.4000/recherchestravaux.733

ISSN : 1969-6434

\section{Éditeur}

UGA Éditions/Université Grenoble Alpes

\section{Édition imprimée}

Date de publication : 28 mai 2015

Pagination : 45-53

ISBN : 978-2-84310-298-1

ISSN : 0151-1874

\section{Référence électronique}

Marie-Claude Hubert, "Beckett : un monde de vieillards », Recherches \& Travaux [En ligne], 86 | 2015, mis en ligne le 28 novembre 2016, consulté le 29 octobre 2021. URL : http://journals.openedition.org/ recherchestravaux/733; DOI : https://doi.org/10.4000/recherchestravaux.733 


\section{Beckett : un monde de vieillards}

Pourquoi y a-t-il tant de personnages vieux chez Beckett? C'est la question à laquelle je vais tenter de répondre ici. Ces personnages ont-ils même fonction dans ce que l'on a pris l'habitude d'appeler son premier théâtre, depuis En attendant Godot jusqu'à Oh les beaux jours, et dans son deuxième théâtre, c'està-dire dans les courtes pièces écrites après?

\section{De pitoyables pantins}

Jusqu'en 1960 environ, c'est un monde de vieillards que Beckett porte à la scène. Les notations concernant leur vieillesse sont nombreuses, tant dans le dialogue que dans les didascalies. Dans En attendant Godot, Vladimir, selon Pozzo, aurait soixante, soixante-dix ans. Estragon a sans doute le même âge, puisque cela fait cinquante ans qu'il partage son existence. Quant à Pozzo qui est chauve et Lucky qui a les cheveux blancs, ils portent ostensiblement les stigmates de la vieillesse. Les quatre personnages de Fin de partie, pièce dans laquelle Beckett représente trois générations, les grands-parents, le père, le fils, ont tous l'aspect de vieillards, même Clov, le plus jeune. Dans Tous ceux qui tombent, Monsieur et Madame Rooney se lamentent sans cesse sur leur vieillesse. Ils ont plus de soixante-dix ans, puisque leur fille, si elle était en vie, aurait une cinquantaine d'années. Monsieur Rooney, dont c'est l'anniversaire, se sent tellement vieux qu'il se demande s'il n'a pas atteint les cent ans. Dans Cendres, Henri parle de sa "vieille viande ${ }^{\mathrm{I}}$ " Krapp, le héros de La Dernière Bande, présenté comme

I. Cendres, précédé de La Dernière Bande, Paris, Minuit, 1959, p. 56. Toute l'œuvre de Beckett étant éditée aux éditions de Minuit, désormais nous n’indiquons plus l'édition. 
« un vieil homme avachi ${ }^{2}$ » a soixante-neuf ans. Maunu, dans Cascando, est lui aussi un vieillard. Seule Winnie, dans Oh les beaux jours, est plus jeune («de beaux restes ${ }^{3}$ ", note Beckett) puisqu' elle a la cinquantaine tandis que son mari a la soixantaine. Jamais aucun enfant n'apparaît dans ce théâtre, sauf le jeune Garçon dans En attendant Godot, mais on ne sait d'où il vient, peut-être de l'au-delà. Quant au «môme» que Clov aperçoit hors du refuge, c'est-à-dire hors scène, il n'est sans doute que le fruit d'une de ses hallucinations.

En raison de leur vieillesse, ces personnages sont souvent diminués, voire infirmes. Certains sont boiteux (Vladimir, Estragon, Clov) ou paralysés (Hamm, Winnie), voire culs-de-jatte (Nagg, Nell), malvoyants (Clov, Winnie) ou aveugles (Pozzo, Hamm). Les pauvres gestes du corps scandent l'action dramatique et suscitent dans leur maladresse un rire amer ${ }^{4}$. Dans En attendant Godot, la démarche chaplinesque de Vladimir ("s'approchant à petits pas raides, les jambes écartées $\left.{ }^{5} »\right)$ qui annonce celle de Clov dans Fin de partie ("démarche raide et vacillante ${ }^{6}$ )) ou de Krapp dans La Dernière Bande ("pas mal assuré $\left.{ }^{»}\right)$ qui glisse sur une peau de banane, la lourdeur d'Estragon («il se lève péniblement, va en boitillant vers la coulisse gauche $\left.{ }^{8} »\right)$, telles qu'elles sont décrites dans les didascalies inaugurales, donnent à la pièce une tonalité clownesque. Vladimir chancelle, titube, tombe lorsqu'il essaie de soulever Pozzo et il lui est ensuite très difficile de se relever. Estragon se plaint dès son entrée en scène d'avoir mal aux pieds et, toujours geignard, il égrène tout au long de la pièce les mêmes lamentations. À maintes reprises, il chancelle lui aussi, perd l'équilibre, manque de tomber. Quant à Pozzo et à Lucky, ils se signalent également par leurs chutes burlesques. À l'acte I, Lucky, attaché à Pozzo, comme un animal en laisse, par une corde qui lui entame le cou, tombe dès que Pozzo tend la corde. Au deuxième acte où Lucky, avec une corde beaucoup plus courte, conduit Pozzo devenu aveugle, lorsque l'un des deux protagonistes s'arrête, l'autre tombe, entraînant le premier dans sa chute. Ils restent à terre, longtemps inertes, si bien que Vladimir et Estragon les croient morts. Les bruits de chute de M. et de Mme Rooney, leurs halètements, dans Tous ceux qui tombent, pièce radiophonique, la reptation malaisée de Willie quand il apparaît au final de Oh les beaux jours, prêtent également à rire tout

2. La Dernière Bande, 1959, p. 7.

3. Oh les beaux jours, I963, p. IO.

4. Nous renvoyons le lecteur à notre ouvrage : Langage et corps fantasmé dans le théâtre des années cinquante. Ionesco, Beckett, Adamov, Paris, José Corti, 1987.

5. En attendant Godot, I952, p. II.

6. Fin de partie, 1957, p. I3.

7. La Dernière Bande, p. 26.

8. En attendant Godot, p. 19. 
comme les jeux dérisoires de Hamm avec ses lunettes d'aveugle, de Clov avec sa longue-vue dans Fin de partie, de Winnie avec sa loupe et ses lunettes dans Oh les beaux jours. Il en va de même de Nagg et de Nell qui répertorient les gestes qui ne leur sont plus possibles, ou qui leur sont devenus difficiles, s'embrasser, se toucher, se tourner l'un vers l'autre, gratter le dos du partenaire.

Cette série de gags clownesques est directement héritée des lazzi de la commedia dell'arte, revus à travers les premiers films comiques, ceux de Chaplin, de Laurel et Hardy ${ }^{9}$, de Keaton ${ }^{10}$ ou des Marx Brothers que Beckett tenait en haute estime. Le corps vieilli, tel qu'on peut le percevoir à la lecture à travers les répétitions incessantes que contiennent les didascalies, a ses rythmes et ses leitmotive. À la représentation, il s'impose en outre dans des images scéniques fortes que le spectateur n'est pas près d'oublier, entrée en scène de Lucky attelé à Pozzo, sa danse chaotique (qu'il appelle, aux dires de Pozzo, "la danse du filet», allusion aux ventes d'esclaves noirs), découverte des têtes de Nagg et de Nell qui émergent des poubelles, apparition au lever de rideau de Winnie, enterrée jusqu'à la taille dans un mamelon de sable, autant d'images qui créent un effet de choc. Donnant à voir l'inéluctable déchéance qui attend tout un chacun au soir de la vie, il est le symbole, saisissant dans le grossissement irréaliste, du lot commun.

Vieillesse est lorsque à croupetons

Crachotant sur les tisons ${ }^{\text {II }}[\ldots]$

Tel est le discours de Musique que Paroles tente de comprendre dans Paroles et Musique, discours dans lequel Beckett parodie «Les Regrets de la Belle Heaumière ${ }^{12}{ }^{2}$, rendant ainsi hommage à la lucidité de Villon face au naufrage de la vieillesse.

\section{Aux portes de la mort}

Dans son deuxième théâtre, Beckett met encore en scène des vieillards, mais, contrairement à ce qu'il faisait jusqu'alors, il n'en décrit pratiquement plus le corps, donnant seulement quelques indications lacunaires pour souligner

9. Rappelons que dans le premier manuscrit de En attendant Godot, Pozzo et Lucky, qui n'ont pas encore de nom, sont appelés, l'un, «le grand», l'autre, "le petit», allusion explicite à Laurel et Hardy.

Io. C'est Buster Keaton que Beckett a choisi comme interprète de Film.

II. Paroles et musique, dans Comédie et actes divers, 1966, p. 6I-78, p. 70.

I2. "Ainsi le bon temps regrettons / Entre nous, pauvres vieilles sottes, / Assises bas, à croupetons, [...] Ainsi en prend à maintes et maintes.» (Le Testament, strophe LVI, dans F. Villon, Euvres poétiques, Paris, Garnier Flammarion, 1965, p. 67.) 
leur extrême vieillesse, toujours les mêmes, notamment la pâleur du visage proche de celle du masque mortuaire et / ou la blancheur des cheveux. Les jeux clownesques ont disparu et avec eux le rire qu'ils faisaient naître. Parvenus tout près de la mort, ces êtres retrouvent confusément des bribes de leur passé. Seulement évoquées par le récit, les images de ce passé ne sont pas montrées. Certes, dans deux des pièces antérieures, le protagoniste se tourne lui aussi vers son passé : Krapp, dans La Dernière Bande, revoit l'année fatidique où mourut sa mère et où il renonça à l'amour pour se consacrer à son œuvre, Winnie dans Oh les beaux jours, se remémore les jours anciens. Mais ni l'un ni l'autre ne se sentent touchés par l'aile de la mort.

Dans Solo ${ }^{\mathrm{r}}$, un Récitant évoque le passé d'un vieillard absent, tandis qu’au fil de son récit, les similitudes entre lui-même et ce dernier se multiplient et qu'il se confond avec lui. Avec leurs " cheveux blancs en désordre ${ }^{14}$ », tous deux sont habillés d'une chemise de nuit blanche et de chaussettes blanches. La chambre du Récitant, avec son lampadaire à pétrole dont le globe blanc imite un crâne - figure de vanité qui vient rappeler l'imminence de la mort -, avec son grabat dans un coin, est identique à celle dans laquelle évolue l'homme dont il est censé raconter l'histoire. Le personnage, un homme qui semble attendre la mort, revoit, dans des flashs très brefs, des images de son enfance. Il évoque les photos, depuis longtemps déchirées et jetées, photos de ses parents qui ont laissé un vide grisâtre sur les murs où elles étaient fixées. À la différence du héros de Film qui regarde les vieilles photos de son passé avant de les déchirer, lui revoit mentalement les images des "êtres chers" : "Là son père. Ce vide grisâtre. Là sa mère. Là tous les deux. Souriants. Jour des noces. Là tous les trois. Cette tache grisâtre. Là tout seul' ${ }^{15}{ }$. Il se rappelle le plus souvent des funérailles et se remémore, comme s'il n'avait jamais connu le jour, les «Années de nuits ${ }^{16}{ }^{\prime}$, l'infinité de nuits vécues depuis la nuit de la naissance, comme si toutes ces nuits dans le souvenir se télescopaient, se confondaient avec celle qu'il est en train de vivre, la dernière sans doute. "Trente mille nuits. Peine à croire si peu $^{17}$.» Le regard distancié porté sur les scènes remémorées donne à entendre le détachement du personnage qui se considère déjà loin de la vie. La sécheresse des phrases très courtes, le plus souvent nominales, dépourvues de déterminants, le recours à des termes cinématographiques («Extrémité du grabat à peine visible au bord du cadre», «Fondu», «Parapluies autour d'une

I3. Pièce composée en 1977.

I4. Solo, dans Catastrophe et autres dramaticules, 1982, p. 26-37, p. 29.

15. Ibid., p. 32.

I6. Ibid., p. 3I.

17. Ibid., p. 30. 
fosse. Vus d'en haut», "cercueil hors cadre, etc. ») accentuent l'impression que le personnage, aux portes de la mort, n'éprouve pas de nostalgie.

Les protagonistes de Cette fois et de Berceuse semblent encore plus proches de la mort que le Récitant de Solo. Comme les mourants qui parfois revoient dans des flashs, de façon accélérée, des instants de leur vie, ils entendent une voix, la leur, évoquer des bribes disparates de leur existence. Dans Cette fois ${ }^{18}$, une tête, celle d'un vieillard assis dans le noir, écoute une voix, la sienne. "Vieux visage blême légèrement incliné en arrière, longs cheveux blancs dressés comme vus de haut étalés sur un oreiller ${ }^{19}$. " La tête, seul point éclairéé ${ }^{20}$, à trois mètres au-dessus de la scène, a un aspect spectral, renforcé par l'opposition saisissante entre le blanc et le noir. Immobile, sauf au final où s'y dessine un sourire, "édenté de préférence ${ }^{21}$ ", qui lui donne un aspect encore plus cadavérique, le visage semble couché sur un lit de mort. Le seul mouvement est celui des yeux, fermés, sauf à quatre reprises lorsqu'ils s'ouvrent après quelques secondes de silence pour se refermer peu après que la voix s'est fait à nouveau entendre afin de se concentrer sur l'écoute. Cloué par les voix du souvenir qui l'assaillent de tous côtés, de gauche, de droite, du dessus, le personnage revoit mentalement le film de sa vie à travers des images chaotiques qui surgissent dans le désordre. Chaque voix cherche à retrouver un moment de la vie du Souvenant ${ }^{22}$. Interrogeant en vain la mémoire, chacune essaie de rappeler le passé, ce que souligne le questionnement des premiers mots de la pièce : «cette fois [...] cette dernière fois [...] quand c'était». Chacune a sa spécificité. Dans la note du premier manuscrit, Beckett indique que chacune doit correspondre aux composantes de la psyché, la voix A étant cognitive, la voix $\mathrm{B}$ mentale, la voix $\mathrm{C}$ affective ${ }^{23}$. Dans la version définitive, la voix $\mathrm{A}$, celle des souvenirs d'enfance, cherche à retrouver le chemin de la vieille ruine où l'homme se réfugiait, enfant, pour lire en cachette, à l'écart de tous. La voix $\mathrm{B}$, celle du souvenir amoureux, tente de ressusciter l'image de la femme

18. Pièce composée en 1974 .

19. Cette fois, dans Catastrophe et autres dramaticules, p. 9.

20. Beckett éprouve lui-même le sentiment qu'il est allé «à l'extrême limite de ce [qui] est possible au théâtre». Il note : "À l'objection que la composante visuelle est trop petite, hors de proportion avec l'auditive, réponse : la réduire encore en vertu du principe que qui peut le moins peut le plus", cité par J. Knowlson, Beckett, Arles, Solin Actes Sud, 1999, p. 759.

2I. Cette fois, p. 25 .

22. Voir W. Asmus, «Rehearsal Notes for the German Premiere of Beckett's That Time and Footfalls at the Schiller-Theater Werkstatt, Berlin (Directed by Beckett)", Journal of Beckett's Studies, nº 2, été 1977 , p. 155 .

23. M. Fehsenfeld, «De la boîte hermétique au regard implacable», Revue d'esthétique Samuel Beckett, Privat, 1986, p. 363-370, p. 365. 
aimée lors des rendez-vous dans la nature, dans un petit bois, sur un chemin de halage, dans les dunes, puis le moment où l'homme s'est retrouvé seul sur ces mêmes lieux. La voix $\mathrm{C}$ est celle qui évoque le vieillard solitaire, qui mène une vie de semi-clochard, lorsque, dans ses déambulations en ville, il cherche à se mettre à l'abri de la pluie, au Musée des Portraits, au bureau de poste ou à la bibliothèque municipale. Les trois récits s'entrecroisent dans un jeu d'échos et de répétitions à la manière des mouvements d'une sonate. Ces tentatives pour redonner vie au passé se soldent par le constat que tout cela n'est que "poussière $\mathrm{e}^{24} »$. Tout est incertain dans le puzzle des souvenirs que l'homme ne parvient pas à reconstituer, pas plus qu'il n'arrive à dire "je», à saisir une unité de son être, ce qui explique sans doute que Beckett le place non au milieu de la scène mais le décentre, comme la femme dans Berceuse. "Une telle précision est peut-être le signe d'une approche qui ne saurait conserver une représentation centrée du sujet, et lui fait subir dans l'espace un déplacement métaphorique de celui qui sera le sien sur la scène excentrique de l'identitées."

Il en va de même dans Berceuse ${ }^{26}$, où une femme, $\mathrm{F}$, assise dans une berceuse, immobile, écoute elle aussi une voix off, la sienne, une voix «blanche, sourde, monotone», lui parler d'elle-même à la troisième personne en lui rappelant le passé. Elle a le "visage blanc sans expression", les «mains blanches serrant les bouts des accoudoirs ${ }^{27}$." Totalement inadaptés à la situation, la « robe du soir », le "bibi incongru, posé de guingois, garni de frivolités», qui contrastent avec «les cheveux gris en désordre», témoignent d'un temps où cette femme, "vieillie avant l'heure", s'adonnait à des mondanités, comme jadis sa mère dont elle porte la robe. Un tel costume laisse entendre que F. a été bien vivante alors qu'elle semble ici tout près de la mort. La voix évoque, en quatre mouvements successifs, les quatre moments qui signifièrent pour elle le retrait de l'existence. Elle rappelle celui où cette femme prit la décision de renoncer au monde extérieur, de cesser de déambuler et de chercher l'âme sœur ("temps qu'elle finisse / finisse d'errer / [...] tout yeux / à l'affût d'un autre / d'un autre comme elle $\left.{ }^{28} »\right)$, celui où elle s'assit, immobile, face à la fenêtre espérant voir son semblable («elle rentra enfin/s'asseoir à sa fenêtre / [...] tout yeux /à

24. Allusion aux paroles de Dieu à Adam dans la Genèse, 3, 19 : "Tu es poussière et tu retourneras en poussière.»

25. D. Soenen, «Le théâtre de la non-coïncidence. Dynamique du paradoxe dans les pièces monologales de Samuel Beckett", Samuel Beckett Today / aujourd'hui, Amsterdam - New York, Sjef Houppermans éd., 2006, p. 379-387, p. 38I.

26. Pièce écrite en 1980.

27. Berceuse, dans Catastrophe et autres dramaticules, p. 39-55, p. 53.

28. Ibid., p. 42. 
l'affût d'un autre $\left.{ }^{29} »\right)$, celui où elle décida de ne plus chercher à voir, et celui où, après avoir baissé le store de la fenêtre, elle descendit s'installer dans la berceuse où mourut sa mère ("se berça / se disant / non / plus jamais ça ${ }^{30}$ »). Ces quatre récits successifs de la voix sont suscités par la femme qui, à quatre reprises, sort de son silence pour dire : «Encore». La voix orchestre le balancement de la berceuse qui commence à bouger chaque fois qu' elle reprend et qui s'immobilise dès qu'elle se tait. Elle se fait berceuse, le mot français permet à Beckett de jouer subtilement de l'ambiguïté du terme qui désigne à la fois une chanson douce et un fauteuil à bascule ${ }^{31}$. Selon les propos de Beckett à Daniel Labeille, «la femme n'impulse en rien le balancement, il est provoqué par le souvenir ${ }^{32}{ }^{2}$. Le renoncement est scandé par le mouvement des yeux qui peu à peu se ferment. Beckett précise dans les didascalies qu'ils sont "tantôt fermés, tantôt grands ouverts» dans le premier mouvement, de plus en plus fermés au cours des deuxième et troisième mouvements, définitivement clos au milieu du dernier. Les étapes du renoncement sont également visualisées par la lumière qui faiblit à chaque arrêt de la voix. L'installation dans la berceuse, qui est celle de la mère, comme l'explique Beckett à Daniel Labeille, marque l'étape finale de ce renoncement. Comme pour Murphy ou pour le personnage de Film, elle est le lieu où s'endormir dans le dernier sommeil. «Le bercement a pour but d'amener le corps à une fin désirée; la chaise doit bercer la femme à mort ${ }^{33}$ [...]». Avec ses «accoudoirs arrondis recourbés comme pour étreindre ${ }^{34}$ », elle signe le lien à la mère. Les retrouvailles mère/fille s'accomplissent là dans une mort semblable au sein de la même berceuse au terme d'une vie identique.

Claudel procédait de façon quelque peu analogue dans ses dernières pièces, Le Livre de Christophe Colomb ou Jeanne d'Arc au bûcher, où il représentait les deux protagonistes revoyant, au seuil de la mort, les moments marquants de leur passé. Déjà entrée dans l'au-delà, Jeanne, attachée au poteau du bûcher, appartient à un monde hors du temps humain tandis que sur le plateau apparaissent dans des flashs les êtres qu'elle a côtoyés dans sa vie passée.

Pour comprendre une vie comme pour comprendre un paysage, il faut choisir le point de vue et il n'en est pas de meilleur que le sommet. Le sommet de la vie de Jeanne d'Arc, c'est sa mort, c'est le bûcher de Rouen. C'est de ce sommet, dans le drame que j'ai écrit pour $\mathrm{M}^{\mathrm{me}}$ Ida Rubinstein avec la collaboration de Honegger,

29. Ibid., p. 45 .

3o. Ibid., p. 52.

3I. Ce que ne permet pas le titre anglais, Rockbaby.

32. E. Brater, Beyond Minimalism: Beckett's Late Style in the Theater, New York and Oxford, Oxford University Press, 1987, p. I73.

33. M. Kedzierski, «Mise en forme de la mort», Europe, $n^{\circ}$ 770-77I, juin-juillet I993, p. 47-52, p. 50.

34. Berceuse, p. 54. 
qu'elle envisage toute la série des événements qui l'y ont conduite, depuis les plus proches jusquaux plus lointains, depuis la consommation jusqu'à l'origine de sa vocation et de sa mission. Ainsi les mourants, dit-on, voient à la dernière heure se déployer tous les éléments de leur vie, à qui sa conclusion imminente confere un sens définitif. Tout de suite, tout est expliqué au regard qui passe d'un horizon à l'autre et du terme au départ ${ }^{35}$.

Harry Halbreich est très admiratif face à un tel procédé de composition :

L'intuition géniale de Claudel a été celle (déjà utilisée dans le Christophe Colomb mis en musique par Darius Milhaud) de la chronologie à rebours, à la manière du flash-back cinématographique, du renversement du temps jusqu'au moment zéro où les présents terrestre et spirituel vont se rejoindre dans le feu dévorant, en cet instant suprême où ceux qui vont mourir remontent par la pensée en un éclair le cours achevé de leur trop brève existence ${ }^{36}$.

La grande différence entre ces dernières pièces de Claudel et celles de Beckett, c'est que chez Claudel, la foi permet au moment de mourir de donner un sens rétrospectif à une vie dont les épisodes se déroulent devant le spectateur tandis que chez Beckett ils n'apparaissent que comme des bribes qui ne constitueront jamais un tout, des bribes qu'aucun sens ne viendra jamais unifier; ils ne sont que confusément évoqués par une voix, celle du protagoniste, voix qui semble même ne plus lui appartenir puisqu'elle lui est extérieure.

Beckett pousse le processus à son extrême limite dans Comédie $^{37}$, où il représente, emprisonnées dans trois jarres identiques qui se touchent, "trois têtes [...] qui restent rigoureusement de face et immobiles d'un bout à l'autre de l'acte. Visages sans âge, comme oblitérés ${ }^{38}$ ", FI, F2, H, l'épouse, la maitresse, le mari. Les trois personnages ne peuvent pas se voir et ne dialoguent pas, chacun ignorant la présence des deux autres à ses côtés. Chacun ne parle que lorsqu'un projecteur, braqué sur son visage, lui extorque la parole pour évoquer alors un passé en lambeaux. Les voix n'ont pas le pouvoir de créer autour d'elles un espace fictionnel dans lequel des actions seraient susceptibles de s'accomplir. Rien ne se passe hic et nunc. Temps, espace, perspective, événement sont ici abolis. Ne subsistent que les trois têtes qui sortent des jarres. Pétrifiés dans leur vase-canope, ces personnages qui habitent un temps sans temps, un espace sans espace, sont des morts vivants. Pour Jean-Jacques Mayoux, «les urnes qui les

35. P. Claudel, Théatre II, Paris, Gallimard, coll. «Bibliothèque de la Pléiade», 1965, «Propos sur un spectacle de ballets", p. I466.

36. H. Halbreich, Arthur Honegger. Un musicien dans la cité des hommes, Paris, Fayard - Sacem, 1992, p. 513.

37. Pièce écrite en 1962.

38. Comédie, dans Comédie et Actes divers, 1966, p. 9. 
contiennent, bien que leurs têtes aux traits corrodés dépassent au-dessus du col, sont des urnes funéraires ${ }^{39}$ ». Gilles Sandier fut d'emblée sensible à cette atmosphère, lui qui souligne le caractère bouleversant du spectacle. C'est «Feydeau vu d'outre-tombe ${ }^{40}$ ». Il se remémore

[...] les trois vases sans âge d'où sortent trois masques bleus et blancs, masques de mort ou de jeux de cartes, trois bouches d'ombre qui remuent les lèvres dans un bruit de glouglou, avant de commencer d'articuler des sons où l'on reconnaît peu à peu des mots, des phrases, des bribes de souvenirs, proférés à une vitesse vertigineuse par des voix détimbrées et monocordes de phonographe ${ }^{41}$.

Adorno retrouve dans la pièce l'atmosphère de la nouvelle de Kafka, Le Chasseur Gracchus, dans laquelle un homme revient du monde des morts hanter de loin en loin les vivants parce qu'il n'a pas trouvé dans l'au-delà de lieu où demeurer, parce qu'il souffre de ne pouvoir vraiment mourir. La tristesse des personnages de Comédie naît elle aussi du sentiment qu'ils n’arrivent pas à mourir.

C'est bien là ce qu'il y a d'extrêmement étrange dans l'idée de Beckett selon laquelle la seule véritable utopie, c'est la mort. Ce vers quoi convergent toutes les aspirations, c'est vers la mort - par opposition à la vie, qui, elle, n'est qu'une souffrance sans fin. [...] Toute l'incroyable énergie de ce poète tourne autour de cet unique point, qu'on peut facilement comprendre ainsi : on ne peut penser le néant, on ne peut l'imaginer autrement que comme le néant de quelque chose. [...] Le thème métaphysique de cette pièce c'est que l'aspiration au néant positif, qui est lié à la banale «histoire triangulaire » complètement désespérée et triste à mourir qu'elle raconte, est désormais refusée aux hommes ${ }^{42}$.

Dans son premier théâtre, Beckett porte à la scène des personnages qui, rendus maladroits par la vieillesse, suscitent le rire, comme les clowns au cirque, rire qui, malgré la distance qu'il introduit, n'exclut pas la pitié car il donne à entendre que la vieillesse est notre horizon à tous. Ensuite, dans son deuxième théâtre, il représente des vieillards proches de la tombe à qui apparaissent les images mentales de leur passé, comme parfois les mourants revoient le film de leur vie, mais ce regard rétrospectif ne leur apporte aucun sens, ne confère au moi aucune unité. C'est cette approche du terme qu'il met en scène dans de courtes pièces comme Solo, Cette fois, Berceuse, lui qui a toujours médité sur les fins dernières. Il va même jusqu’à nous donner à voir cette proximité du terme, juste avant le grand saut dans l'inconnu, dans Comédie. C'est tout cela qui donne à son théâtre une dimension métaphysique.

39. J.-J. Mayoux, "Samuel Beckett, homme de théâtre», Biblio (numéro spécial Beckett), Hachette, janvier 1967, p. I5-2I.

40. G. Sandier, Arts, Io juin 1964.

4I. G. Sandier, Théâtre et combat. Regards sur le théâtre actuel, Paris, Stock, I970, p. 5 I.

42. T. W. Adorno, Notes sur Samuel Beckett, Caen, Nous, 2008, p. I06-I07. 